\title{
Examining the Prevalence of Previously Recorded Phenotypically Related Diagnoses Among Fee-for-Service Medicare Enrollees Newly Diagnosed with Mendelian Conditions
}

J Gen Intern Med 37(2):475-7

DOI: $10.1007 / \mathrm{s} 11606-020-06469-8$

(C) Society of General Internal Medicine 2021 limiting to those categories because black and white race constituted the majority of cases.

We had IRB and Centers for Medicare and Medicaid Services (CMS) approval to conduct this work through CareJourney's Virtual Research Data Center.

\section{RESULTS}

In 2019, of 39,917,598 beneficiaries fully enrolled in fee-forservice Medicare between 2016 and 2019, 28,377 had a newly coded diagnosis of at least 1 of the Mendelian diseases that we studied (Table 1). Polycythemia vera was the most common, representing $44 \%$ of all conditions examined; achondroplasia was the least common. With the exceptions of hereditary hemochromatosis and polycythemia vera, older white women accounted for most cases.

Depending on the disorder, between 60.5 and $87.8 \%$ of patients with a newly diagnosed Mendelian disorder in 2019 had at least 5 phenotypically related diagnoses in the previous year; between 73.6 and $97.3 \%$ had at least 5 phenotypically related diagnoses coded across the previous 3 years (Table 2). In 2018 , between 29.7 and $58.7 \%$ of patients newly diagnosed in 2019 had at least 10 phenotypically related diagnoses; aggregating data from 2016 to 2018 , those proportions grew to between 50.4 and $84.8 \%$.

\section{DISCUSSION}

We used 4 years of Medicare fee-for-service data to identify beneficiaries with any of 12 newly coded Mendelian diseases and examined the prevalence of phenotypically related diagnoses in the 3 preceding years' billing records. For a given condition, up to $87.8 \%$ of identified patients had 5 or more related diagnoses in the year immediately preceding a new Mendelian condition diagnosis; up to $97.3 \%$ had 5 or more across the preceding 3 years.

Our findings suggest that - even in the older, Medicareinsured population that we studied - artificial intelligenceinformed decision support might help providers identify patients with Mendelian disorders by aggregating constellations of diagnoses recorded in the recent past that suggest an overarching one. ${ }^{3}$ Surprisingly, a not insubstantial

Received October 1, 2020

Accepted December 13, 2020

Published online January 21, 2021 

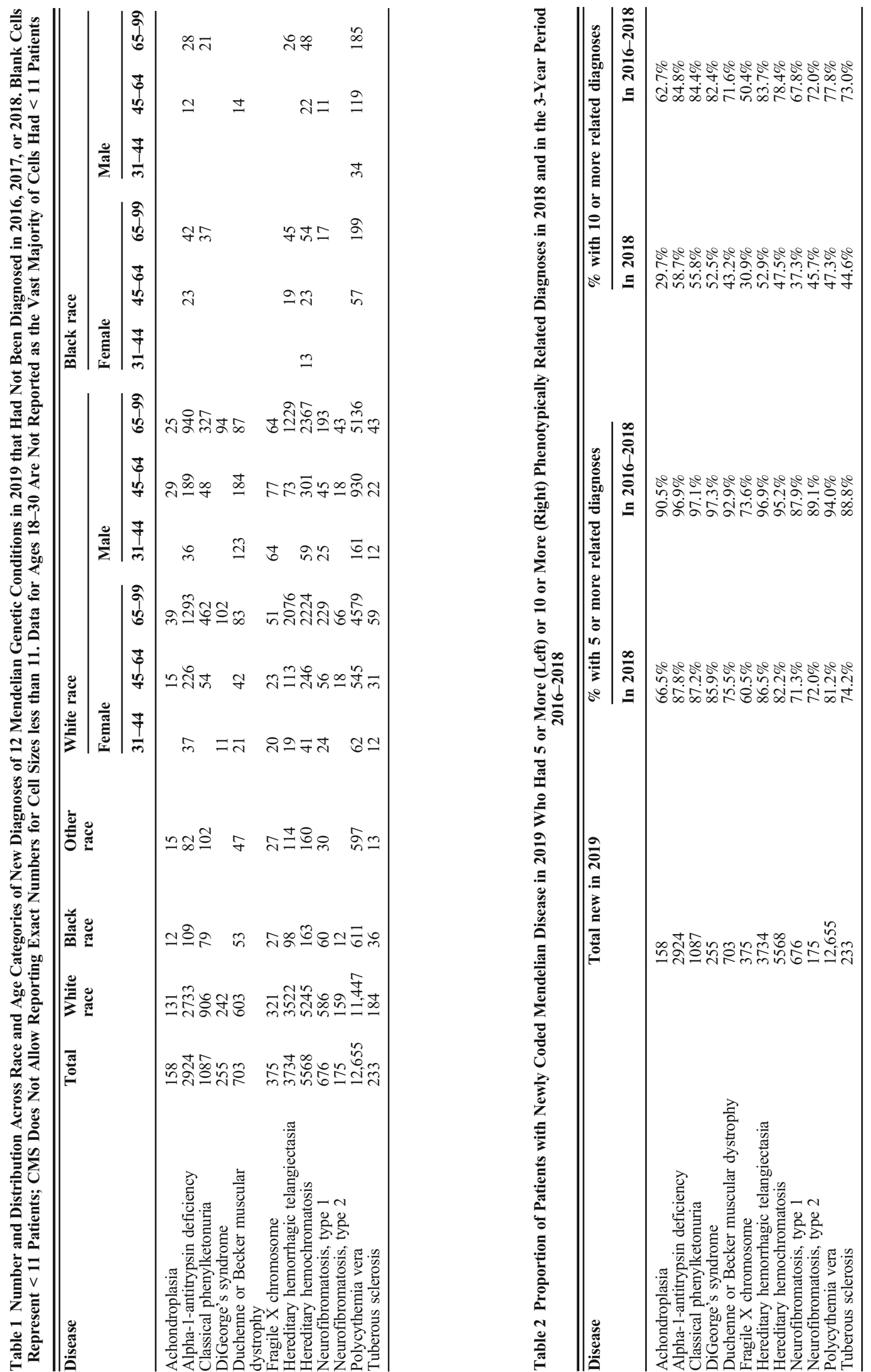
number of Medicare beneficiaries might be identified with the disorders we studied.

Our study has several limitations. First, it is possible that Mendelian disorder diagnostic codes were simply not recorded for 3 years before reappearing in 2019. While we recently found a fairly dramatic year-to-year drop-off in diagnostic coding of chronic conditions, ${ }^{6}$ Mendelian conditions tend to be life-long, disabling, and, frequently, visually apparent; it should be somewhat surprising for them not to be recorded. Second, we were not able to confirm the diagnoses we studied with genetic testing, as Batarache et al. were able to do. ${ }^{2}$ Third, our study was limited by its reliance on relatively recent administrative datasets wherein final reconciliation delays might trivially impact dataset completeness. Finally, future research should explore whether artificial intelligence-based decision support using recent phenotypically related diagnoses is appropriate for the Medicare-insured population.

Nonetheless, our analysis demonstrates that there are relatively large numbers of individuals in the fee-for-service Medicare beneficiary population that might be identified as having a Mendelian genetic disorder by screening phenotypically related diagnostic billing codes. Among those for whom the diagnosis is indeed novel, earlier genetic testing and diagnosis of these Mendelian disorders might lead to better treatment and outcomes.

William B. Weeks, $M D, P h D, M B A^{1}$

Grace Huynh, $M D, P h D^{1}$

Stacey Y. Cao, $\mathrm{MHS}^{2}$

Jeremy Smith, $M P H^{3}$

Chaitanya Bangur, $P h D, M B A, M S^{1}$

James $N$. Weinstein, DO, MS ${ }^{1,4,5,6}$

${ }^{1}$ Microsoft Corporation, Microsoft Research, Redmond, WA, USA

${ }^{2}$ CareJourney, Arlington, VA, USA
${ }^{3}$ White River Junction VA Outcomes Group, WRJ, Hartford, VT, USA

${ }^{4}$ The Dartmouth Institute, Lebanon, NH, USA

${ }^{5}$ Kellogg School of Business,

Evanston, IL, USA

${ }^{6}$ Amos Tuck School of Business, Hanover, NH, USA

Corresponding Author: William B. Weeks, $M D, P h D, M B A$; Microsoft Corporation, Microsoft Research, Redmond, WA, USA (e-mail: william.weeks@microsoft.com).

\section{Compliance with Ethical Standards:}

Conflict of Interest: The authors declare that they do not have a conflict of interest.

CareJourney has IRB approval ("Developing Care Model, Provider and Network Evaluation Tools using Medicare Claims and Assessment" Solutions IRB Study \#2019, CMS DUA 52882) to analyze CMS data through a Virtual Research Data Center.

\section{REFERENCES}

1. Bastarache L, Hughey JJ, Goldstein JA, et al. Improving the phenotype risk score as a scalable approach to identifying patients with Mendelian disease. J Am Med Inform Assoc. 2019;26:1437-47.

2. Bastarache L, Hughey JJ, Hebbring S, et al. Phenotype risk scores identify patients with unrecognized Mendelian disease patterns. Science. 2018;359:1233-9.

3. Dias R, Torkamani A. Artificial intelligence in clinical and genomic diagnostics. Genome Med. 2019;11:70.

4. Wu P, Gifford A, Meng X, et al. Mapping ICD-10 and ICD-10-CM Codes to Phecodes: Workflow Development and Initial Evaluation. JMIR Med Inform. 2019;7:e14325

5. Phenome Wide Association Studies Resources. Human Phyotype Ontology to ICD10 maps. https://phewascatalog.org/. Accessed July 9, 2020.

6. Weeks WB, Huynh G, Cao SY, Smith J, Weinstein JN. Assessment of year-to-year patient specific comorbid conditions reported in the Medicare Chronic Conditions Data Warehouse. JAMA Netw Open. 2020;3(10): e2018176.

Publisher's Note: Springer Nature remains neutral with regard to jurisdictional claims in published maps and institutional affiliations. 